\title{
T2* Mapping with Background Gradient Correction Using Different Excitation Pulse Shapes
}

N.M. Hirsch and C. Preibisch

\section{ABSTRACT}

SUMMARY: Background gradients induced by magnetic susceptibility variations near air-filled cavities in the brain cause signal-intensity loss in gradient-echo images and shorten $\mathrm{T} 2^{*}$ considerably. With a correction method in which the exponential decay is restored with section-profile-dependent correction factors, parts of the signal intensity can be recovered. While uncorrected T2* values drop by $20 \%$ at a gradient strength of $75 \mu \mathrm{T} / \mathrm{m}$, with correction and exponential excitation pulses, this boundary is pushed to $220 \mu \mathrm{T} / \mathrm{m}$.

A ccurate $\mathrm{T}^{*}$ measurement is important for a variety of applications. In Parkinson disease studies, it is used to gain insight into the iron content in the substantia nigra. ${ }^{1}$ It is also required for a quantitative analysis of the oxygen distribution in the brain. ${ }^{2}$ The sensitivity of $\mathrm{T} 2{ }^{*}$ to variations in oxygen saturations is exploited in functional activation studies. ${ }^{3}$

Local macroscopic magnetic susceptibility differences, particularly near air-tissue interfaces, can severely reduce $\mathrm{T} 2{ }^{*}$ values. Especially at high field strengths, image quality is degraded in large parts of the brain.

Recently, a method has been described for correcting the signal intensity for deviations from monoexponential decay by using exponential excitation pulse shapes. ${ }^{4}$ The deviation of the signal intensity from undisturbed exponential decay is corrected with the help of prefactors calculated from the temporal profile of the excitation pulse and the simultaneously measured local background gradient strength. This correction extends the information content of the $\mathrm{T}^{*}$ map in the affected areas, yet at the price of a more imprecise section profile.

The aim of the current study was to compare 3 different pulse

Received November 11, 2011; accepted after revision December 16.

From the Departments of Neuroradiology (N.M.H., C.P.) and Neurology (C.P.), Technische Universität München, Munich, Germany.

This work was supported by the Deutsche Forschungsgemeinschaft SFB824. N.M.H. is supported by Deutsche Telekom Stiftung, the Free State of Bavaria, and the Graduate School of Information Science in Health.

Paper previously presented in part at: Annual Meeting of the European Society for Magnetic Resonance in Medicine and Biology, October 6-8, 2011; Leipzig, Germany; and Annual Meeting of the Deutsche Gesellschaft für Neuroradiologie, October 6-8, 2011; Köln, Germany.

Please address correspondence to Nuria Hirsch, Abteilung für Neuroradiologie, Klinikum rechts der Isar der TU München, Ismaninger Str 22, 81675 München; email: nuria.hirsch@tum.de

shapes (sinc-Gauss, sinc, and exponential) and to determine the susceptibility gradient strength up to which $\mathrm{T} 2{ }^{\star}$ mapping yields reliable results.

\section{TECHNIQUE}

With an excitation pulse time profile $A$ and a section-selection gradient strength $G_{\mathrm{s}}$, the resulting signal intensity in the presence of a background susceptibility gradient $G_{\text {susc }}$ is described by ${ }^{4,5}$

$$
S(T E) \propto A\left(\frac{G_{\text {susc }}}{G_{s}} \cdot T E\right) \cdot e^{-T E / T 2^{*}},
$$

where $G_{\text {susc }}$ is assumed to be linear and perpendicular to the selected section. Thus, the signal-intensity decay is not necessarily exponential any more but rather influenced by the temporal shape of the pulse. The weighting, in turn, depends on the susceptibility gradient strength as well as on the section-selection gradient in a way that is different for each pulse shape.

A gradient map in the through-plane direction is required to obtain the correction factor $1 / A$. For a distance between voxels $\Delta x$, the gradient strength $G_{\text {susc }}$ is obtained from the phase difference $\Delta \phi$ of the first and second echoes ${ }^{4}$ :

$$
G_{\text {susc }}=\frac{\Delta \phi}{\gamma \cdot \Delta x \cdot\left(T E_{2}-T E_{1}\right)} .
$$

Across a thicker section, a given susceptibility gradient effects a larger range of magnetic field values and thus signal intensity loss, which is why the correction method generally works better for thinner sections. The phase map was calculated from real and imaginary data acquired simultaneously with the magnitude data.

Exponential and sinc-shaped time profiles were defined as ${ }^{5}$

$$
A_{\text {expo }}(t)=\exp \left(-4 \frac{\left|G_{\text {susc }}\right|}{G_{\text {s }}} \cdot \frac{t}{p / 2}\right),
$$

and 

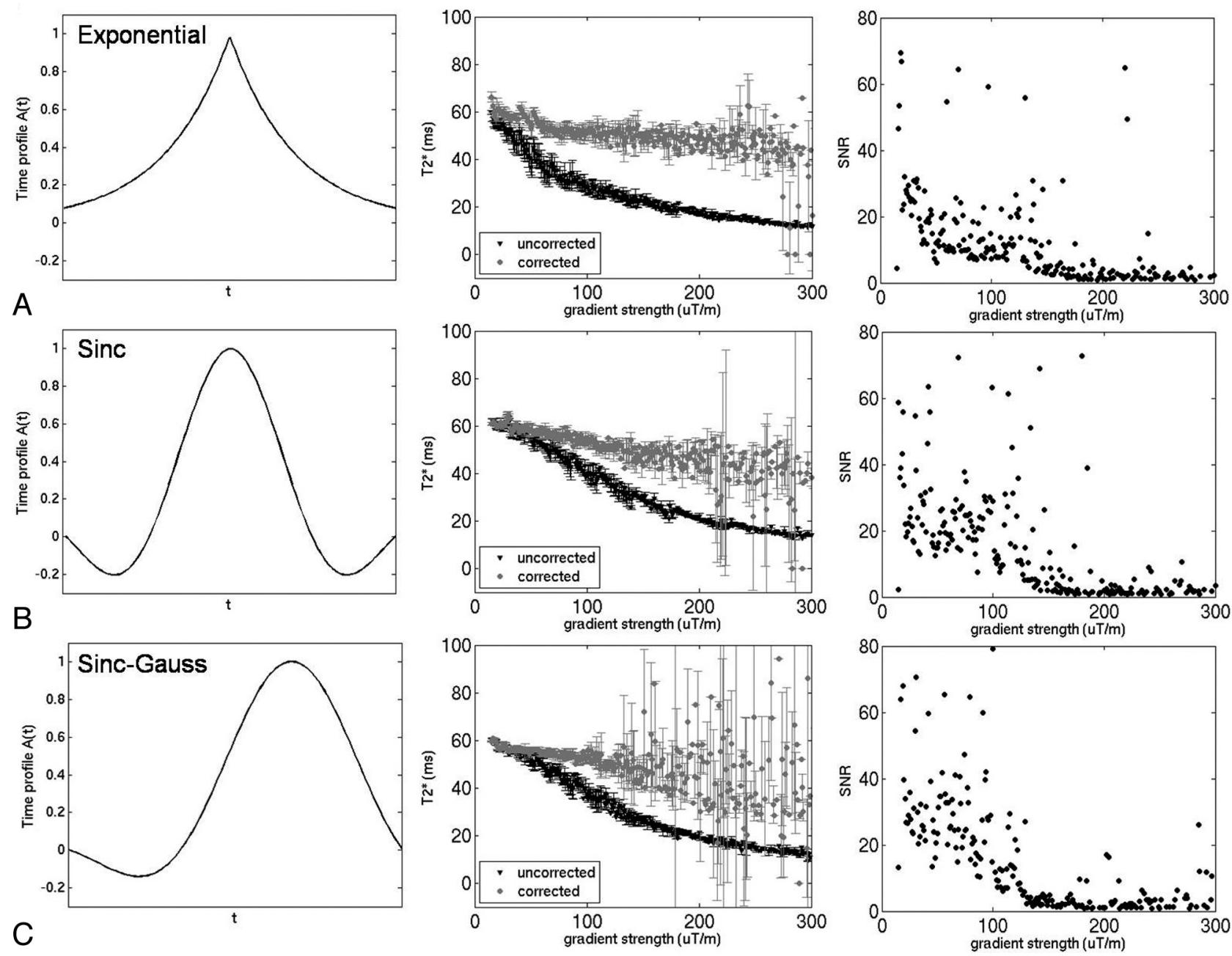

FIG 1. Left column: graphic representations of the pulse shapes. Center column: $T 2 *$ values of a homogeneous phantom at different gradient strengths. Error bars indicate the SD of different T2* values measured in voxels experiencing the same gradient strength. Right column: SNR at different gradient strengths ( $T E=48 \mathrm{~ms}$ ). Row $A$, Exponentially shaped pulse. Row B, Sinc-shaped pulse. Row $C$, Sinc-Gauss-shaped pulse.

4)

$$
A_{\text {sinc }}(t)=\operatorname{sinc}\left(2 \pi \frac{\left|G_{\text {susc }}\right|}{G_{\mathrm{s}}} \cdot \frac{t}{p / 2}\right) \cdot \cos \left(\frac{\pi}{2} \frac{\left|G_{\text {susc }}\right|}{G_{\mathrm{s}}} \cdot \frac{t}{p / 2}\right),
$$

with a pulse duration $p($ Fig $1 A,-B)$. The cosine term acts as a filter to achieve a smooth excitation spectrum. The third pulse with an optimized sinc-Gauss shape, which is used by default in fast-fieldecho imaging protocols, was approximated numerically (Fig 1C).

Magnitude, real and imaginary images in transverse orientation were acquired with a $3 \mathrm{~T}$ Achieva scanner (Philips, Best, the Netherlands) by using an 8-channel head coil. A multi-gradientecho sequence with 10 evenly spaced echoes was used to acquire 40 sections $\left(\mathrm{TE}_{1}=3 \mathrm{~ms}, \Delta \mathrm{TE}=5 \mathrm{~ms}, \mathrm{TR}=2055 \mathrm{~ms}\right.$, rapid flyback, $\mathrm{FOV}=184 \times 132 \mathrm{~mm}^{2}$, in-plane resolution $=2 \times 2$ $\mathrm{mm}^{2}$, section thickness $=3 \mathrm{~mm}$, reconstructed matrix $=112 \times$ 112, flip angle $=60^{\circ}$, total scan time $=3$ minutes, 5 seconds). Apart from the scanner-default sinc-Gauss-shaped excitation pulse, sinc or exponentially shaped pulses could be selected. The respective values for the pulse duration $p$ and the section selection gradient $G_{\mathrm{s}}$ were $2.11 \mathrm{~ms}, 11,289 \mu \mathrm{T} / \mathrm{m}$ (sinc-Gauss), $2.78 \mathrm{~ms}$, and $11,274 \mu \mathrm{T} / \mathrm{m}$ (sinc and exponential). Imaging was performed on a spherical homogeneous gel phantom consisting of $1.75 \%$ agar dissolved in a $0.9 \%$ sodium chloride solution doped with 0.11 $\mathrm{mmol} / \mathrm{L}$ of gadolinium diethylene triamine pentaacetic acid. Although in vivo susceptibility gradients are primarily due to air cavities, similar gradient strengths in the phantom can be induced by using a small piece of metal positioned at the bottom of the phantom. Additionally, images of 4 volunteers were acquired ( 3 men, $32.5 \pm 15.2$ years of age).

The weighting of the exponentially decaying data with the pulse profile was eliminated by using correction factors according to equations 3 and 4 and the numerically approximated sincGauss shape. A voxelwise monoexponential fit of the corrected data was then performed to obtain $\mathrm{T}^{*}$ maps. All calculations were done in Matlab 7.9 (MathWorks, Natick, Massachusetts).

\section{DISCUSSION}

In Fig $1, \mathrm{~T}^{*}$ values are plotted against the corresponding susceptibility gradient strengths for a cuboid volume of interest in the phantom where the background gradient exhibits strong variation over several sections. Uncorrected $\mathrm{T} 2{ }^{*}$ values drop by $20 \%$ at a gradient strength of $75 \mu \mathrm{T} / \mathrm{m}$ for sinc-shaped pulses, $63 \mu \mathrm{T} / \mathrm{m}$ for sinc-Gauss-shaped pulses, and $34 \mu \mathrm{T} / \mathrm{m}$ for exponential pulses. Data acquired with the sinc-Gauss-shaped pulse can be 


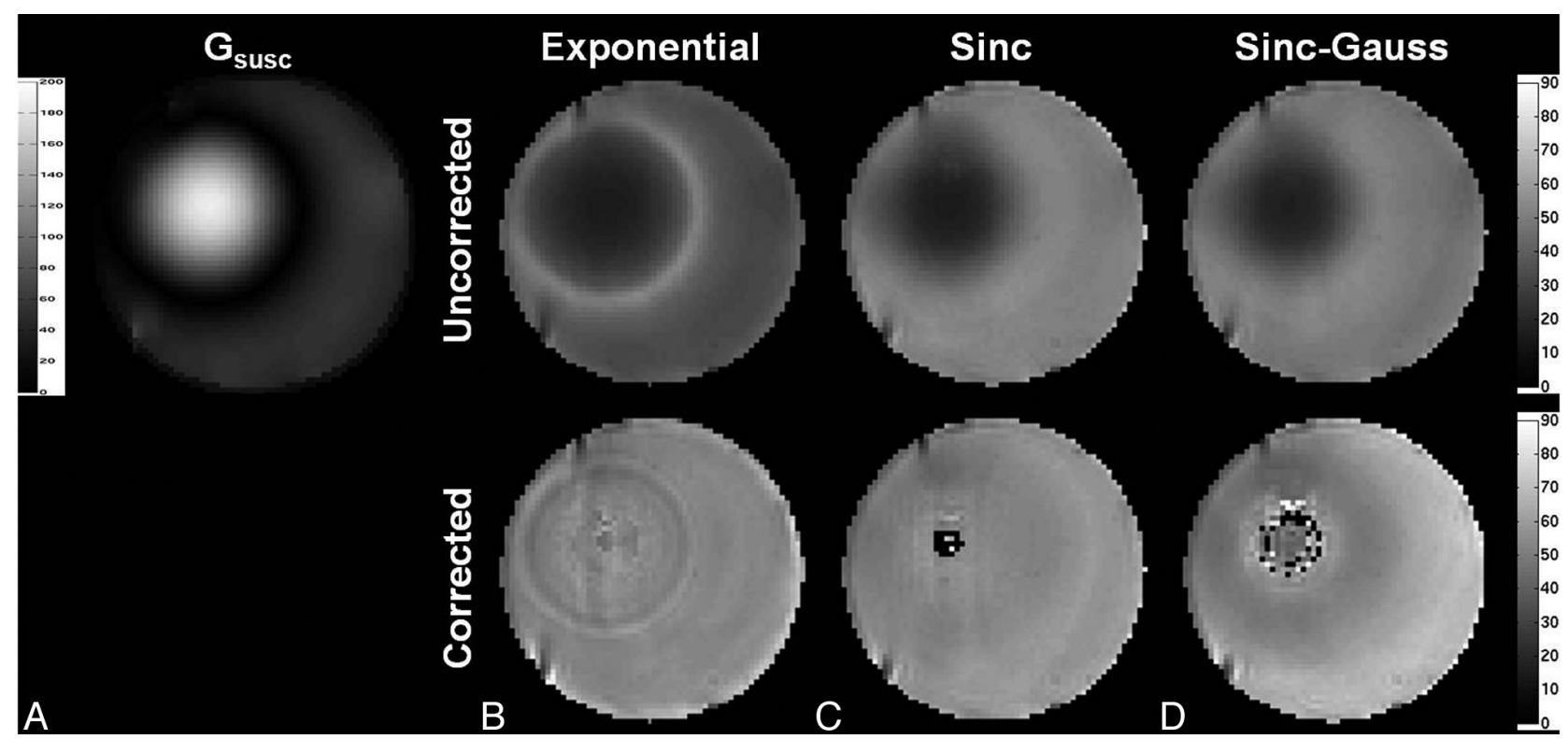

FIG 2. A, Susceptibility gradient map. Also shown are uncorrected (top) and corrected (bottom) T2* maps of a section of a homogeneous phantom with exponential $(B)$, sinc $(C)$, and sinc-Gauss $(D)$ excitation pulses.

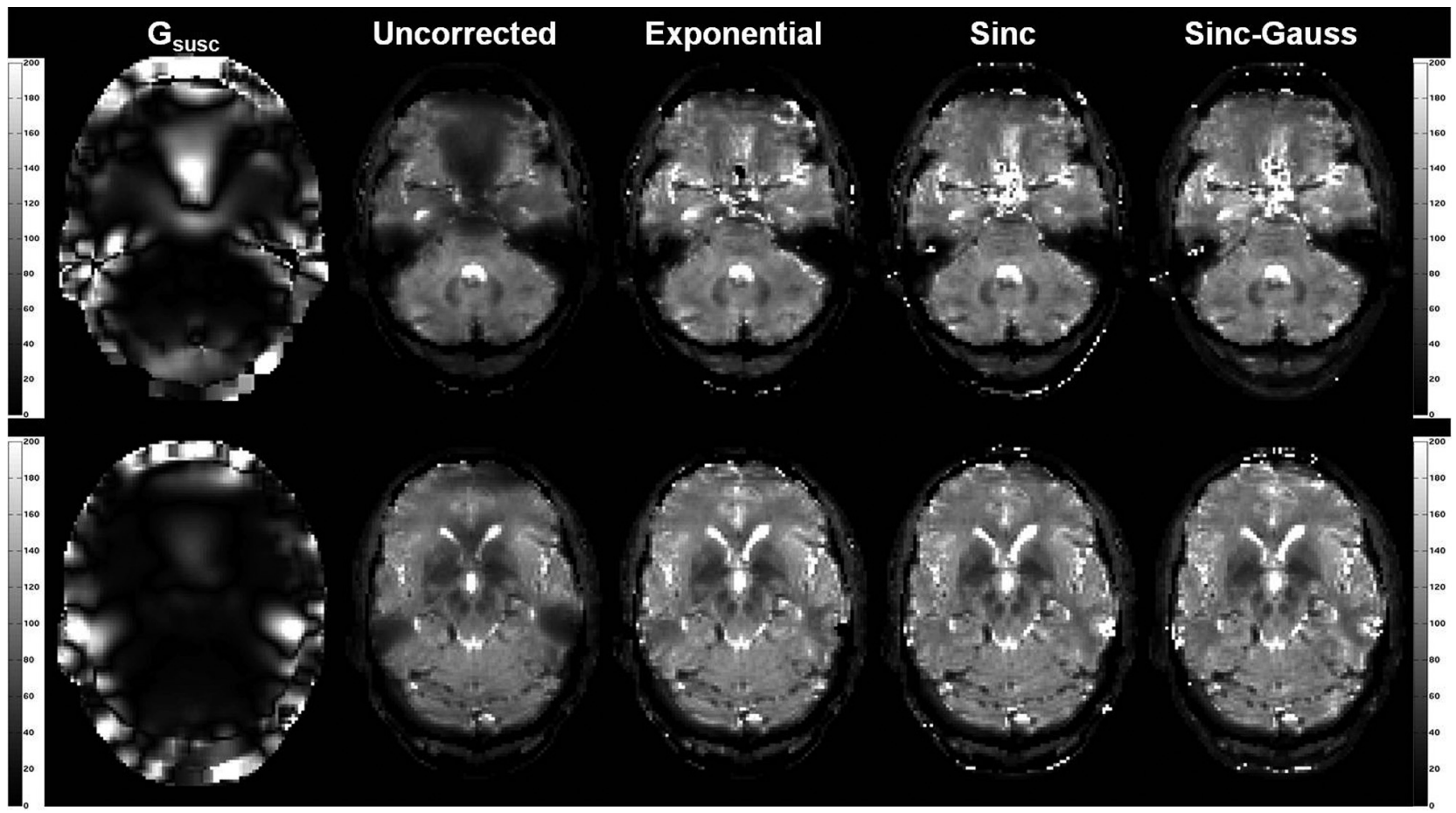

FIG 3. In vivo $\mathrm{T}^{*}$ maps of 2 sections. From left to right: smoothed through-plane gradient map; uncorrected $\mathrm{T}^{\star}$ map acquired with an exponential pulse; corrected maps with exponential, sinc, and sinc-Gauss pulses.

corrected with the applied method for gradient strengths up to approximately $100 \mu \mathrm{T} / \mathrm{m}$ with a deviation of $<20 \%$. In the presence of stronger background gradients, the resulting $\mathrm{T} 2{ }^{*}$ values are not reliable. With the same $20 \%$ criterion, data acquired with the sinc-shaped pulse can be corrected up to gradients of $175 \mu \mathrm{T} / \mathrm{m}$, whereas for exponentially shaped pulses, the $20 \%$ boundary is reached at $220 \mu \mathrm{T} / \mathrm{m}$. With exponential pulses, even higher field gradients can be corrected to some extent.

Corrected values spread more than uncorrected values due to the additional source of uncertainty when a measured susceptibility gradient map is used. Also shown in Fig 1 is the SNR at different gradient strengths at TE $=48 \mathrm{~ms}$. SNR was calculated as the mean of the signal intensity at a given susceptibility gradient strength divided by its SD. For each of the pulses, SNR drops significantly at gradient strengths where corrected $\mathrm{T} 2^{\star}$ values start to spread and become unreliable. At low gradients, SNR is higher for sinc and sinc-Gauss pulses than for exponential pulses, indicating that such pulses might be the better choice in this case. 
Figure 2 shows the effect of the different pulse shapes on phantom $\mathrm{T} 2 *$ maps. Compared with the other 2 pulse shapes, an exponentially shaped pulse enhances susceptibility effects in the uncorrected $\mathrm{T} 2^{\star}$ map, because the exponent of the time profile represented in equation 3 is added to the exponent of the pure $\mathrm{T} 2{ }^{*}$ decay in equation 1 , therefore lowering the effective time constant of the decay. However, data acquired with the exponential pulse can be corrected for higher susceptibility gradient strengths when the sinc and the sinc-Gauss corrections have already failed. Still, if only very weak background gradients interfere with the measurement, a sinc-shaped pulse with its more rectangular section profile can be better corrected for. When using exponential pulses, a sufficient section gap and interleaved acquisition mode should be chosen.

The superior performance of exponentially shaped pulses can be explained as follows: The time profile of the numerically optimized sinc-Gauss pulse can only be approximated in the correction routine, whereas the analytic representation of the other 2 pulses is accurate. Both the sinc-Gauss and the sinc-shaped pulses show zero crossings in their time profiles, which result in increased signal-intensity losses compared with the exponential pulse. From equations 1, 3, and 4, and the numeric representation of the sinc-Gauss profile, critical values for $G_{\text {susc }}$ can be determined where the signal intensity has dropped to a certain value. At $\mathrm{TE}=48 \mathrm{~ms}$, an $80 \%$ signal intensity loss is reached at susceptibility gradients of $131 \mu \mathrm{T} / \mathrm{m}$ (exponential), $43 \mu \mathrm{T} / \mathrm{m}$ (sinc), and 118 $\mu \mathrm{T} / \mathrm{m}$ (sinc-Gauss), respectively.

From the analysis of phantom data, it can be seen that a true plateau cannot be maintained for any of the tested pulse shapes. However, the information in the $\mathrm{T} 2{ }^{\star}$ map that is accessible for diagnostic evaluation can be extended considerably by using an exponentially shaped excitation. Especially regions close to airfilled cavities in the orbitofrontal cortex or in the temporal lobes now become visible for inspection, even though the absolute values might not be quite accurate (Fig 3).

At $3 \mathrm{~T}$, typical gradient values of $300-500 \mu \mathrm{T} / \mathrm{m}$ occur in extended areas. ${ }^{5,6}$ We observed in vivo background gradients up to approximately $400 \mu \mathrm{T} / \mathrm{m}$. With the help of exponential pulses and a correction routine that accounts for the modified $\mathrm{T} 2^{\star}$ decay as described above, large parts of the $\mathrm{T}^{\star}{ }^{\star}$ map can be recovered, even at such field variations, contrary to the other tested pulse shapes. Exponentially shaped pulses thus appear to be favorable for in vivo measurements. Their somewhat more imprecise Lorentzian section profile can be compensated sufficiently by choosing an interleaved acquisition scheme and a small gap between sections.

Disclosures: Nuria Hirsch—RELATED: Grant: Free State of Bavaria, Comments: scholarship for the duration of the PhD, Support for Travel to Meetings for the Study or Other Purposes: Graduate School for Information Science in Health.

\section{REFERENCES}

1. Baudrexel S, Nurnberger L, Rub U, et al. Quantitative mapping of T1 and $\mathrm{T} 2{ }^{\star}$ discloses nigral and brainstem pathology in early Parkinson's disease. Neuroimage 2010;51:512-20. Epub 2010 Mar 6

2. An H, Lin W. Quantitative measurements of cerebral blood oxygen saturation using magnetic resonance imaging. J Cereb Blood Flow Metab 2000;20:1225-36

3. Howseman AM, Thomas DL, Pell GS, et al. Rapid T2* mapping using interleaved echo planar imaging. Magn Reson Med 1999;41:368-74

4. Baudrexel S, Volz S, Preibisch C, et al. Rapid single-scan T2*-mapping using exponential excitation pulses and image-based correction for linear background gradients. Magn Reson Med 2009;62:263-68

5. Preibisch C, Volz S, Anti S, et al. Exponential excitation pulses for improved water content mapping in the presence of background gradients. Magn Reson Med 2008;60:908-16

6. Weiskopf N, Hutton C, Josephs O, et al. Optimized EPI for fMRI studies of the orbitofrontal cortex: compensation of susceptibilityinduced gradients in the readout direction. MAGMA 2007;20: $39-49$ 